\title{
Reflexiones sobre la paz mundial
}

\section{Situación Actuat}

\section{Gontrol de armas por superioridad neta}

Las dos superpotencias están oscilando actualmente entre dos visiones estratégicas, cuya predominancia se alterna de acuerdo a las tendencias políticas internas y a la percepción que cada una tiene de la conducta de la otra.

El primer punto de vista enfatiza la imposibilidad final de una victoria militar en la era nuclear. Todo conflicto importante se volverá nuclear, cualquiera sea su lugar de origen y su forma inicial. Y cualquier conflicto nuclear importante traerá consigo el exterminio mutuo de los superpoderes. La única solución, por lo tanto, es preservar la paz y, para hacerla posible, adoptar políticas y medidas apropiadas para supervisar adecuadamente el control mutuo de armas, tales como SALT I y SALT II.

El segundo punto de vista recalca que los avances tecnológicos y otras medidas, tanto ofensivas como defensivas, pueden proporcionar una superioridad neta a alguno de los superpoderes. Los avances tecnológicos significan, o bien un primer ataque exterminador por sorpresa (hoy inconcebible), o bien medidas defensivas importantes y efectivas (hoy también inconcebibles).

Entre las condiciones que pueden llevar a una superioridad neta, además de un avance tecnológico, está la desestabilización de la economía y, consecuentemente, de la estructura sociopolítica de uno de los superpoderes (como en el caso de la Unión Soviética, de acuerdo a algunos expertos norteamericanos), inducida por el peso excesivo de las inversiones militares. Otro conjunto de condiciones desestabilizadoras puede ser la pérdida del suficiente consenso, nacional e internacionalmente, con sus efectos paralizantes, frente a las políticas de uno de los superpoderes (como en el caso de los Estados Unidos, de acuerdo con algunos expertos soviéticos)

Una tercera posibilidad sería una "guerra nuclear limitada" peleada en Europa, que trajera consigo una "victoria de teatro" que, si bien definiría la supremacía del ganador, no destruiría el área 
esencial de ninguno de los superpoderes ni destruiría el régimen interno ni la capacidad nuclear del perdedor.

Una cuarta posibilidad sería una "guerra espacial", por la cual uno de los superpoderes trataría de destruir, en el espacio, los satélites y otras naves espaciales del enemigo, adquiriendo por lo tanto una superioridad neta para sus capacidades de vigilancia y de prevención. Como esta hipótesis es todavía tecnológicamente remota, no se volverá a discutir en este trabajo.

Cualesquiera sean los medios por los cuales uno de los superpoderes alcance una superioridad estratégica neta, de acuerdo a los. que apoyan este punto de vista, la consecuencia sería la aceptación obligada de las imposiciones del más fuerte por parte del poder más débil, o su derrota militar final, en el caso de una confrontación importante.

\section{El estancamiento resultante}

La oscilación de los superpoderes entre los dos puntos de vista estratégicos conflictivos anteriormente mencionados, significa un mayor riesgo de guerra. Puesto que el control de armamentos jamás se cumple en forma estable $y$ perdurable en ambos superpoderes, en cada uno de ellos tiende a ser sucedido por frenéticos períodos de armamentismo, bajo la presunción real o imaginaria (como ahora, con el gobierno de Reagan), de que el superpoder comprometido quedaría estratégicamente rezagado frente al otro y necesitaría ponerse al día urgentemente. Tales períodos de rearme de parte de uno de los superpoderes son percibidos inmediatamente por el otro, como una amenaza inaceptable, llevándolo a renovar sus esfuerzos de rearme.

De las condiciones actuales pueden extraerse, por consiguiente, dos conclusiones. La primera es que la interrupción de los esfuerzos para controlar los armamentos tiene, en último término, el efecto de agravar la carrera armamentista. La segunda conclusión; ampliamente reconocida por los expertos, es que un proceso continuo de rearme que comprenda: 1) una reducción aún más crítica del lapso que cada uno de los superpoderes tiene para certificar si el otro está lanzando o no un ataque nuclear y, al mismo tiempo, 2) la automatización autoguiada cada vez mayor de respuestas de contragolpe, aumenta muchísino la posibilidad de una guerra occidental (co mo ha sido demostrado por muchas falsas alarmas electrónicas), de manera que parece ser imposible escapar a ella en un futuro no remoto.

Más aún, se debe recalcar que los puntos de vista sobre la eventual desestabilización de alguno de los superpoderes, cualquiera sean las posibilidades de que se produzca tal resultado, contienen una gran falacia en su propio nivel conceptual. Ella consiste en 
suponer que un superpoder afectado por crecientes procesos de desestabilización soportaría pasivamente tal proceso. Inversamente, existen todas las razones para pensar que el prospecto de su propia desestabilización constituiría una amenaza inaceptable para cualquiera de los superpoderes, induciéndolos a igualar tal amenaza con la amenaza de la agresión nuclear, con todos los efectos de convertirse en un aliciente para la guerra.

En el caso de una guerra nuclear limitada, las perspectivas son más obscuras. El superpoder perdedor experimentaría la fuerte conveniencia de aceptar un arreglo con el ganador para limitar sus pérdidas y para preservar, junto con su integridad territorial, las posibilidades de mantener, por una parte, su propio régimen interno $y$, por la otra, su capacidad nuclear básica.

Sin embargo, de acuerdo con circunstancias impredecibles, que incluyen las poco razonables exigencias del ganador, el poder perdedor puede verse obligado a intentar obtener ventajas compensatorias, destruyendo algunos blancos estratégicos en el núcleo territorial del ganador. En tal caso, una represalia del ganador podría desatar una confrontación nuclear mayor.

\section{El balance de terror}

El Tratado SALT II, firmado en Viena el 18 de junio de 1979, es el último intento para alcanzar una estabilización relativamente general de la carrera armamentista, fundamentada en el principio de mantener una paridad estratégica entre los superpoderes. E1 Tratado no excluyó nuevos desarrollos tecnológicos estratégicos, pero por Io menos, pretendió impedir nuevos desarrollos cuantitativos. Se estableció un tope de 2.400 lanzamientos por cada grupo, previendo una limitación cuantitativa de 2.250, empezando el 31 de diciembre de 1981, y también se adoptaron otras prohibiciones.

El Tratado, al que se opusieron fuertemente los conservadores en los Estados Unidos y que todavía no ha sido ratificado por el Congreso y el Gobierno de Reagan, si bien se observa todavía en la práctica (también lo hacen los soviéticos), ha propuesto una nueva formulación (START). Reagan afirma que los soviéticos, en los últimos años, han invertido muchísimo más que los Estados Unidos en defensa estratégica (se dice que en 1982 fueron us $\$ 273$ billones contra us\$195 por parte de los Estados Unidos) y que sus 2.498 plataformas de lanzamientos estratégicos sobrepasan significativamente la capacidad americana, con 1.918 .

La controversia concerniente al escenario europeo tiene lugar en torno a lo que se refiere a este desequilibrio, cuya falacia es la omisión de los aspectos cualitativos de los armamentos en cuestión. La "opción cero" de Reagan presenta a los soviéticos una alternativa: - bien la NATo recobra su balance de poder a través del despliegue 
de 572 nuevos misiles en Europa (108 Pershings x y 464 misiles de crucero) o Ios soviéticos aceptan desmantelar 333 de sus ss-20, que están en todo el territorio soviético, como también 280 de sus anticuados misiles ss-4 y ss-5.

Los soviéticos rehusaron esa propuesta, principalmente en vista de la capacidad nuclear preexistente de Francia y Gran Bretaña. A cambio, proporcionaron algunas indicaciones de que podrían retirax 380 plataformas de lanzamiento de Europa, con 580 cabezas nucleares y desmantelar sus ss-4 y ss-5, manteniendo, sin embargo, 155 ss-20 como contrapeso a los 160 misiles franceses y británicos.

En un intento para alcanzar un punto de vista más objetivo acerca del relativo poder estratégico de los dos bloques en Europa, el último Informe Anual (1982) del Instituto Francés de Relaciones Internacionales indica (p. 37), basado en una experta combinación de diversos datos oficiales, las cifras referentes a los puntos de vista americanos y soviéticos sobre el equilibrio de fuerzas en el escenario europeo, presentados en el cuadro de página 8.

\section{NEGESIDAD DE UN NUEVO ENFOQUE}

\section{Imperativo de una nueva acción}

Gualquiera evaluación honesta y objetiva de la actual situación hace surgir dos conclusiones: 1) la guerra será prácticamente inevitable antes de fin de siglo, e incluso en el curso de esta década, si no se adopta un nuevo enfoque consistente para consolidar la paz; 2) tal enfoque requiere, al mismo tiempo, una movilización seria, a nivel internacional, de otros grupos legítimos además de los superpoderes $y$, en el terreno interno, de todo el estrato social. Además, requiere de una comprensión global de los problemas mundiales, que vaya más al fondo y abarque materias más amplias que aquellas que sólo se relacionan con el control de armamentos.

\section{Los requerimientios de paz}

El requerimiento básico para una paz mundial estable y duradera es la inexistencia de condiciones, para cualquier país o grupo de países, que les permitan usar la violencia internacional como un medio individual creíble para lograr sus propias metas. La inexistencia de condiciones para el uso internacional de la violencia es una situación que se puede lograr, ya bien por una gran difusión de los medios para la violencia internacional o por la concentración de tales medios bajo el control de una autoridad internacional efectiva y representativa.

La amplia difusion de los medios para la violencia internacional, tal como ocurrió en Europa durante la Edad Media, no previene la

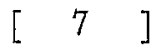




\section{PERSPECTIVA DE LOS EE.UU.}

U.S.

URSS

Misilos

TOTAL

SS-20......... . 250

SS-4/5 - . . . . . . . 350

SS-12/22 . . . . . . . 100

SS-N-5 . . . . . . . 50

Bombarderos

F-111 en Europa Occidental . 164 Backfire . . . . . . . . 45

FB-11l en USA . . . . . 63 Blinder Badger . . . . . 350

$\mathrm{F}-4$. . . . . . . . . . . $265 \mathrm{SU}-17$

A6/A7 (en el mar) . . . . 68 SU_-24

Mig-27 . . . . . . . . 2.700

U.S. Y NATO

Misiles

Francés tierra-tierra . . . . . 18 SS-20 . . . . . . . . . . . 243

Francés mar-tierra . . . . . . 80 SS-4/5 . .. . . . . . . . 253

Inglés estilo Polaris. . . . . . 64 SS-N/5 . . . . . . . . . . 18

TOTAL

I62

514

Bombarderos

F-111 . . . . . . . 72 Backfire

FB-1I1 . . . . . . . 65 Badger

F-4... . . . . . . 46 Blinder . . . . . . . 461

$\mathrm{A} 6 / \mathrm{A} 7$. . . . . . . 40

Mirage (Exancia) . . . . . 46

Vulcan (Gr. Bretaña) . . . . 55 
existencia de guerras locales sino que convierte en muy improbable, empíricamente, una guerra mundial. La otra alternativa es más comprehensiva. La sumisión de los principales medios para la vio. lencia internacional al control de una autoridad internacional efectiva y representativa, eliminaría los riesgos de guerras hasta donde esa autoridad se mantenga efectiva y realmente internacional.

En las condiciones contemporáneas, la amplia difusión de los medios para la violencia internacional no parece ser una meta posible o deseable. Sin embargo, los argumentos en contra de la "difusión" re las capacidades nucleares no son estrictamente correctos y llevan impreso el interés de los superpoderes. Si fuese realmente posible alcanzar un estado de amplia difusión de las capacidades nucleares. creíble para todas las partes involucradas, lo que es actualmente una imposibilidad práctica, se preservaría mejor la paz mundial. Como en una visión contemporánea de la Edad Media, ninguna nación ni grupo de naciones sería capaz de imponer su voluntad individual sobre el mundo como un todo. Sin embargo, es obvio que sería im. posible actualmente alcanzar esa difusión creíble. Más aún, la analogía de la Edad Media no debería ser llevada demasiado lejos porque, en vista de los devastadores efectos de los artefactos nucleares en las condiciones contemporáneas, incluso en las guerras locales, tendría los efectos inaceptables de genocidio, sin hablar de la tendencia a la propagación.

$\mathrm{La}$ alternativa de una autoridad internacional efectiva y verda. deramente representativa se mantiene, por lo tanto, como la única alternativa valiosa. En último término esa autoridad constituye el camino confiable por el cual puede ser mantenida una paz mundial estable y duradera.

La idea de una regulación internacional que someta a todos los estados del mundo a un orden internacional legal, bajo el legitimo control de una autoridad internacional, es a la vez antigua y la única solución realmente racional diseñada hasta ahora para tal propósito. La "Perpetual Peace", de Kant (1975) y "The Problem of the War and the Ways to Peace", de Bobbio (1979), pueden ser mencionadas como las dos referencias convencionales, antiguas y recientes, sobre el tema.

En vista de tal suposición básica, deben considerarse dos asuntos principales. El primero, de carácter teórico, se refiere a los requerimientos analíticos básicos para la constitución y preservación de una autoridad internacional representativa, capaz de mantener bajo control los principales medios para la violencia internacional. El segundo, de carácter más práctico, se refiere a los requerimientos básicos para una transición de la situación actual del mundo hacia una en la cual pueda ser establecida y mantenida tal autoridad internacional. 


\section{Requerimientos básicos para una autoridad internacional}

Una autoridad territorial representativa y efectiva resulta de la combinación de la concentración de un poder político real sobre un territorio dado, apoyado por la posesión, por parte de tal poder, de una legitimidad ampliamente reconocida.

Históricamente, los estados modernos se han originado en Europa desde fines de la Edad Media, por la integración bajo diversas formas (predominantemente por la violencia) - promovida por un agente territorial o dinástico- de autoridades feudales preexistentes que gozaban de diversos grados de autonomía dentro de estados unificados más amplios, principalmente mediante la incorporación de pueblos de la misma cultura en un estado nacional. Posteriormente los nuevos estados más grandes, preferentemente en su calidad de estados nacionales, adquirieron creciente representatividad a través de la universalización de la legitimidad democrática.

En las condiciones en que se encuentra el mundo contemporáneo, el establecimiento de una autoridad internacional efectiva y representativa requeriría la transferencia real a tal autoridad de los más altos niveles de poder político, por parte de los estados nacionales, de acuerclo con procedimientos ampliamente reconocidos como legitimos, al menos entre los principales poderes mundiales.

La autoridad internacional tendría que consistir en un sistema colectivo, integrado por delegados renovables, para poder sèr representativos de las realidades políticas mundiales. Sería necesario que este sistema diferenciara una rama legislativa de una ejecutiva y una judicial, para que así se adapte a la estructura formal del proceso político contemporáneo.

Por otra parte, la autoridad internacional necesitaría tener atribuciones de carácter confederativo o superfederativo. Desde Kant, es obvio que el mundo contemporáneo no puede ser regulado y gobernado internacionalmente en la forma de un estado superunitario. Incluso si no es probable que algunos, o una mayoría, de los actuales estados nacionales vayan a tener una vida muy larga, sobrevivirán por un tiempo relativamente largo como las agencias territoriales reguladoras de los asuntos locales. Por lo tanto, un acuerdo superfederativo o confederativo parece ser el camino adecuado a través del cual pueden ser regulados los intereses internacionales comunes de la humanidad, comprendiendo cosas tales como la mantención efectiva de la paz, la preservación de la ecosfera, las regulaciones de problemas de salud y de sistemas internacionales vitales $\mathrm{o}$, en el dominio de la economía, los temas de comunicación, transporte, etc.

Es prácticamente imposible y de poco valor en las actuales condiciones, incluso en una forma especulativa, tratar de averiguar las formas por las cuales se puede llevar a cabo la constitución de una 
autoridad internacional efectiva y representativa, y los acuerdos internacionales por los cuales puede ser regulada. Los precedentes históricos analógicos concernientes a la formación de estados modernos o de imperios mundiales, son sólo de una eficacia limitada. El uso predominante de la violencia en la formación de los estados nacionales modernos de Europa no puede ser extrapolado a las condiciones actuales. Hasta cierto punto, la formación de dos bloques de poderes mundiales, como consecuencia final de la Segunda Guerra Mundial y bajo el liderazgo respectivo de los Estados Unidos y la. Unión Soviética, representa los límites conceptuales y empíricos de la posibilidad de extrapolaciones históricas. La inviabilidad de una solución militar, como ya se indicó en este breve estudio, impone precisamente un límite a la validez de ciertas experiencias históricas. Por lo tanto, un procedimiento consensual es un requerimiento necesario, en las actuales condiciones internacionales, para la formación de una autoridad internacional, inclusive si tal procedimiento comprendería la participación activa de sólo un limitado número de los principales poderes mundiales. Se podría discutir la manexa cómo llegar a esa meta, pero sólo en una fase histórica mucho más avanzada del proceso.

\section{SOBRE LAS CONDICTONES DE LA TRANSIGIÓN}

\section{Situación actual}

El actual orden mundial internacional se caracteriza por el hecho de ser el resultado de un balance inestable y variado de la interacción de alrededor de 160 estados formalmente independientes, que persiguen sus intereses nacionales en la arena internacional, de acuerdo a sus respectivas autopercepciones y autodeterminaciones. Tal orden es inestable, porque no se subordina a una legitima norma internacional, y porque no es la expresión de un equilibrio de poder suficientemente estable entre los participantes.

Los estados que interactúan en la arena mundial presentan niveles muy distintos de capacidades efectivas y se someten a condiciones básicas precisas. Se destaca, entre ellos, su gruesa diferenciación en tres "mundos": el "Primer Mundo", incluyendo las sociedades industriales, la mayoría pertenecientes al Hemisferio Norte y reguladas por una economía de mercado básicamente libre, integran como un todo el "bloque americano"; el "Segundo Mundo", que incluye a una variedad de estados, muchos de los cuales presentan altos niveles de industrialización, regulados por economías centralmente controladas y que está integrado como bloque en el "bloque soviético"; el "Tercer Mundo", que incluye una variedad de estados mayor aún, algunos cercanos a la industrialización total, donde la mayoría de ellos son todavía sociedades agrarias, sometidas en for- 
ma diversa a economías de mercado o centralizadas may a menudo bajo gobiernos autoritarios, tienen en común algunas de las características básicas del subdesarrollo. Aquellos estados tienden al no alineamiento, vis a vis los dos bloques. Sin embargo, algunos de esos estados son miembros del Tercer Mundo y se alinean ya bien con el bloque soviético, como Guba, o con el americano, como Honduras.

Los factores que inducen la participación de algunos de esos estados en uno de los dos bloques de poder en competencia; son muy diversos para cada bloque, además de las diversidades a nivel de los estados individuales.

Hablando aproximadamente, el bloque americano está integrado por sociedades donde una mayoría de la gente desea esa asociación, porque piensa que conviene a su sistema económico, que corresponde a sus principales valores y que, desde su punto de vista, representa una condición esencial para su seguridad internacional.

También hablando aproximadamente, el bloque sọxiético está integrado por sociedades organizadas bajo el control aùtoimpuesto de un partido único, que profesa oficialmente una ideología marxista-leninista y donde una élite partidaria autoimpuesta, reproducida por procedimientos cooptativos cerrados, es intimamente dependiente, por una parte, de la continua observancia de la ideología $y$, por otra, del apoyo externo de la Unión Soviética.

Como ya se indicó, a pesar de su inestabilidad, el actual orden mundial internacional manifiesta una fuerte tendencia a mantener sus principales características, tales como los "tres mundos" ya mencionados, el equilibrio estratégico entre dos bloques de poder y el dominio de cada centro imperial sobre su propia periferia.

La elasticidad de tal orden inestable se debe a dos factores principales. Uno es el hecho de que los superpoderes gozan de las condiciones necesarias para preservar su propia primacía. El segundo factor es que los países que integran los dos bloques de poder - contribuyendo así al mantenimiento de un equilibrio de poder no regulado- ya bien tienden a considerar tal integración como necesaria para la preservación de sus intereses fundamentales (como en el caso de la NATO) o bien se someten a un régimen de control político dependiente de tal integración (como en el: caso del Pacto de Varsovia).

\section{Tendencias al cambio}

No obstante la fuerte elasticidad del actual orden internacional, hay importantes factores de cambio que están operando en él.

-Algunos de estos factores están conectados con acontecimientos dentro del Tercer Mundo. El rompimiento de China con la Unión Soviética y'su continuo desarrollo a través de un camino autónomo 
constituye un importante factor de cambio. El desarrollo total de algunos grandes países del. Tercer Mundo, especialmente Brasil, México e India, a pesar de sus obstáculos temporales tales comö sus problemas financieros actuales, está creando nuevos poderosos actores internacionales, inclinados inherentemente a un camino autónomo.

Sin embargo, el más importante factor de cambio lo constituyen los eventos que tienen lugar en los dos bloques de poder. En el bloque americano, el evento más importante es la conciencia, que crece con rapidez en Europa Occidental, de que su territorio se ha convertido en el principal teatro de cualquier confrontación nuclear limitada. En el bloque soviético, además de una conciencia equivalente en lo que se refiere a los europeos orientales, existe una conciencia adicional de que sus respectivos desarrollos nacionales requieren un cambio sustancial en sus regímenes y en su relación con los soviéticos.

El estancamiento estratégico entre los dos superpoderes los hà inducido, al estar cada vez más alejados de un avance tecnológico efectivo, a considerar maneras de preservar sus propios territorios de grandes devastaciones que, en último término, traerían consigo el exterminio recíproco. En vista de ello, los dos superpoderes han estado considerando la hipótesis de una limitada guerra nuclear, la cual necesariamente se efectuaría en Europa. Tal guerra tendría un ganador. En tal hipótesis, el perdedor tendería a aceptar, dentro de condiciones relativamente tolerables, la supremacía del ganador, si bien manteniendo sus propias características internas y sus propias capacidades nucleares. Tal hipótesis representa una verión moderada viable de "victoria militar", sin la consecuencia de la aniquilación mutua.

Los europeos comprenden - cada vez más lejos de un improbáble desenvolvimiento tecnológico o de una perspectiva ahora remota de paz estable- que una guerra limitada en Europa, con su consiguiente devastación, se ha convertido en una condición para que los superpoderes prevengan su exterminio mutuo.

Esta conciencia está haciendo que la primera reacción de los europeos occidentales sea la de rehusar la instalación de nuevos misiles en sus países. Sin embargo, el desarrollo de tal conciencia traerá, inevitablemente, una revisión total de parte de Europa Occiden. tal de todas sus relaciones con el sistema de la NATO, como también de sus relaciones generales con Ios Estados Unidos.

Igualmente, los europeos del Este están cada vez más preocupados con el desarrollo de la estrategia de la guerra limitada, con Europa como teatro de operaciones. Más aún, la mayoría de los europeos orientales tienen la convicción de que sus regímenes domésticos necesitan cambios sustanciales, que comprenden sistemás de producción más eficientes y una representatividad democrática efec- 
tiva, tanto en términos políticos como industriales. El problema es cómo manejar estos asuntos en un sistema cerrado, en el cual la estabilidad de la ideología es una base necesaria para la estabilidad de los regimenes y de las autoridades y en los cuales las cadenas que unen los diversos partidos comunistas y sus líderes a Moscú son tanto un requisito para la preservación del poder, a nivel nacional como el instrumento de dependencia, vis a vis los soviéticos.

\section{La salida}

En las condiciones ya indicadas cexiste una línea de acción realista capaz de conducir al mundo en dirección de una paz estable mientras sea todavfa posible prevenir una gran guerra?

Mi argumento es que tal línea de acción puede ser concebida e implementada en forma realista. Para ello, primero hay que reconocer, de acuerdo con las consideraciones precedentes de este estudio que, en último término, sólo una autoridad internacional verdaderamente efectiva y representativa dispondrá de las condiciones para reforzar una paz estable. En segundo lugar, se debe reconocer que cualquier cambio en el actual estancamiento estratégico debe ser iniciado por aquellos actores que no sean los superpoderes, $Y$ uno debe comprender finalmente que aquellos posibles actores, en las condiciones actuales, son los europeos, particularmente los europeos occidentales -quienes probablemente iniciarán el proceso- y entre ellos, los alemanes.

Por las razones ya indicadas, la conversión de los superpoderes a la idea de una guerra limitada que necesariamente tendría Europa como escenario, ha creado allí una reacción creciente, que tiende a dominar en todos los partidos y a prevalecer en todos los principales sectores sociales. Por consiguiente, es probable que se detenga el despliegle de nuevos misiles en Europa y que, a continuación, el movimiento europeo para la paz, convertido en una tendencia bipartidaria, revise toda la concepción de la defensa europea y de los patrones de asociación de los europeos con los superpoderes.

Para los europeos, la alternativa básica es la neutralización de Europa Occidental y Oriental de forma similar a la pasada neutralización de Austria. La neutralización de Europa presentaría la ventaja, además de su inmensa contribución a la paz, de percaitir la disolución negociada y concomitante de los dos pactos militares en confrontación: NATO y Varsovia. Retirándose de los dos pactos, los europeos podrán reforzar su integración económica, tanto en Europa del Este como del Oeste, con crecientes lazos cooperativos entre las dos mitades.

La neutralización de Europa no significará simplemente la exclusión de la hipótesis de una guerra limitada, con sus consecuencias para Europa como teatro de operaciones. . Crearía directa e in- 
directamente condiciones extraordinariamente favorables para la recuperación y prosperidad económica de toda el área, incluyendo Europa Oriental. Ello haría viable dentro de ciertas condiciones, o bien la reunificación de Alemania o, por lo menos, una cooperación estable y estrecha entre los dos estados alemanes. Del mismo modo haría posible, en condiciones aceptables para los soviéticos, la adopción de cambios grandes y profundamente deseados en los regímenes de Europa Oriental, concediéndoles una autonomía sustancialmente mayor, vis a vis los soviéticos. Y por último, contrariamente a lo que dicen Ias actuales advertencias de los militares, tal proceso realzaría extraordinariamente la fuerza política de Europa, convirtiéndola en un nuevo e importante interlocutor inteinacional, exigiendo el apoyo del Tercer Mundo.

La creación de esas nuevas condiciones haría ponerse en movimiento nuevas y poderosas fuerzas en dirección a una paz confiable. Los dos superpoderes, reduciclos a una confrontación estratégica que ya no es compartida por el resto del mundo y que otra vez comprende las consecuencias inevitables de su aniquilación mutua, experimentariaa fuertes incentivos para alcanzar un orden mundial estable. Y así se crearían las condiciones básicas para un proceso pacífico de orden por medio de la coexistencia internacional, orientado en último término al establecimiento de una autoridad internacional legítima y efectiva. 